\title{
Functional finishing in cotton fabrics using zinc oxide nanoparticles
}

\author{
A YADAV, VIRENDRA PRASAD, A A KATHE, SHEELA RAJ, DEEPTI YADAV, \\ C SUNDARAMOORTHY and N VIGNESHWARAN* \\ Nanotechnology Research Group, Central Institute for Research on Cotton Technology, \\ Mumbai 400 019, India
}

\begin{abstract}
Nanotechnology, according to the National Nanotechnology Initiative (NNI), is defined as utilization of structure with at least one dimension of nanometer size for the construction of materials, devices or systems with novel or significantly improved properties due to their nano-size. The nanostructures are capable of enhancing the physical properties of conventional textiles, in areas such as anti-microbial properties, water repellence, soil-resistance, anti-static, anti-infrared and flame-retardant properties, dyeability, colour fastness and strength of textile materials. In the present work, zinc oxide nanoparticles were prepared by wet chemical method using zinc nitrate and sodium hydroxide as precursors and soluble starch as stabilizing agent. These nanoparticles, which have an average size of $40 \mathrm{~nm}$, were coated on the bleached cotton fabrics (plain weave, 30 s count) using acrylic binder and functional properties of coated fabrics were studied. On an average of $75 \%$, UV blocking was recorded for the cotton fabrics treated with $2 \% \mathrm{ZnO}$ nanoparticles. Air permeability of the nano-ZnO coated fabrics was significantly higher than the control, hence the increased breathability. In case of nano-ZnO coated fabric, due to its nano-size and uniform distribution, friction was significantly lower than the bulk-ZnO coated fabric as studied by Instron ${ }^{\circledR}$ Automated Materials Testing System. Further studies are under way to evaluate wash fastness, antimicrobial properties, abrasion properties and fabric handle properties.
\end{abstract}

Keywords. Friction; nanoparticles; tensile strength; UV-blocking; zinc oxide.

\section{Introduction}

Nanotechnology is concerned with materials whose structures exhibit significantly novel and improved physical, chemical, and biological properties, phenomena, and functionality due to their nanoscaled size (Wang 2000). Metal nanoparticles have received attention in recent years because of their potential applications in microelectronics (Andres et al 1996), photo catalysis (Kamat 2002), magnetic devices (Thomos 1988) and powder metallurgy (Perenboom et al 1981). The intrinsic properties of a metal nanoparticle are mainly determined by size, shape, composition, crystallinity and morphology (Dickson and Lyon 2000). A method for the preparation of platinum nanorods and self-assemblies of nanorods using reduced silicotungstate-based composite at room temperature is demonstrated (Shanmugam et al 2005).

With the advent of science and technology, a new area has developed in the realm of textile finishing. Nanocoating the surface of textiles, clothing, and textiles for footwear is one approach to the production of highly active surfaces to have UV-blocking, antimicrobial and self-cleaning properties. The self-cleaning property can be imparted by nano- $\mathrm{TiO}_{2} /$ nano- $\mathrm{ZnO}$ coating (Lamb et al 2004), while

\footnotetext{
*Author for correspondence (nvw75@yahoo.com)
}

nano-Ag will impart antimicrobial property. Nanoparticle coating may affect the other fabric properties like dyeing property, strength, bending rigidity and air permeability. Fabric friction, which is defined as the resistance to motion, can play a crucial role in the textile industries. Here, the friction is associated with the cutting of fabric multilayer and the separation of the fabrics in garment industries, and the friction of garments on other garments, upholstery and press covers. The tetrapod-shaped $\mathrm{ZnO}$ whisker was used to improve the antistatic property of polyacrylonitrile (PAN) fibre (Jin et al 2004). Another study (Das et al 2005) reported that the frictional force increases as the cellulose fibre component increases.

Earlier, our research group synthesized and characterized the $\mathrm{ZnO}$ nanoparticles using solid-state reaction (Prasad et al 2006). In this work, the $\mathrm{ZnO}$ nanoparticles were prepared by a novel aqueous method and applied onto cotton fabrics using acrylic binder. The UV-blocking property of these fabrics was evaluated in addition to their friction and mechanical properties.

\section{Experimental}

\subsection{Synthesis of $\mathrm{ZnO}$ nanoparticles}

The zinc oxide $(\mathrm{ZnO})$ nanoparticles were prepared by wet chemical method using zinc nitrate and sodium hydroxide 
as precursors and soluble starch as stabilizing agent. Different concentrations of soluble starch $(0 \cdot 1 \%, 0 \cdot 5 \%$, and $1 \%$ ) were dissolved in $500 \mathrm{~mL}$ of distilled water by using microwave oven. Zinc nitrate, $14.874 \mathrm{~g}(0 \cdot 1 \mathrm{~mol})$, was added in the above solution. Then the solution was kept under constant stirring using magnetic stirrer to completely dissolve the zinc nitrate. After complete dissolution of zinc nitrate, $0.2 \mathrm{~mol}$ of sodium hydroxide solution was added under constant stirring, drop by drop touching the walls of the vessel. The reaction was allowed to proceed for $2 \mathrm{~h}$ after complete addition of sodium hydroxide. After the completion of reaction, the solution was allowed to settle for overnight and the supernatant solution was then discarded carefully. The remaining solution was centrifuged at $10,000 \times \mathrm{g}$ for $10 \mathrm{~min}$ and the supernatant was discarded. Thus obtained nanoparticles were washed three times using distilled water. Washing was carried out to remove the byproducts and the excessive starch that were bound with the nanoparticles. After washing, the nanoparticles were dried at $80^{\circ} \mathrm{C}$ for overnight. During drying, complete conversion of $\mathrm{Zn}(\mathrm{OH})_{2}$ into $\mathrm{ZnO}$ takes place.

\subsection{Characterization of $\mathrm{ZnO}$ nanoparticles}

The UV-visible spectra of $\mathrm{ZnO}$ suspended in deionized water were recorded in Specord 50 ANALYTIKJENA ${ }^{\circledR}$ spectrophotometer, from $200-800 \mathrm{~nm}$. The sample was sonicated prior to measurement, for uniform dispersion. The powder X-ray diffraction was performed using Phillips ${ }^{\circledR}$ PW 1710 X-ray Diffractometer with nickel filtered $\mathrm{CuK} \alpha$ ( $\lambda=1.54 \AA$ ) radiation and analysed using APD (Automatic Powder Diffraction) software. The diffracted intensities were recorded from $30-80^{\circ} 2 \theta$ angles.

\subsection{Coating of cotton fabrics with $\mathrm{ZnO}$ nanoparticles}

A fine-medium weight $100 \%$ cotton woven fabric (plain weave, $75.30 \mathrm{~g} / \mathrm{m}^{2}$; ends, 75/inch; picks, 60/inch) was used for the application purpose. $\mathrm{ZnO}$ nanoparticles were applied on cotton using pad-dry-cure method. The cotton fabric cut to the size of $30 \times 30 \mathrm{~cm}$ was immersed in the solution containing $\mathrm{ZnO}(2 \%)$ and acrylic binder (1\%) for $5 \mathrm{~min}$ and then it was passed through a padding mangle, which was running at a speed of $15 \mathrm{~m} / \mathrm{min}$ with a pressure of $15 \mathrm{kgf} / \mathrm{cm}^{2}$ to remove excess solution. A $100 \%$ wet pick-up was maintained for all of the treatments. After padding, the fabric was air-dried and then cured for $3 \mathrm{~min}$ at $140^{\circ} \mathrm{C}$. The fabric was then immersed for $5 \mathrm{~min}$ in $2 \mathrm{~g} / \mathrm{l}$ of sodium lauryl sulfate to remove unbound nanoparticles. Then the fabric was rinsed at least 10 times to completely take out all the soap solution. The fabric thus washed was air-dried. Simultaneously, bulk-ZnO coating was carried out for comparison.

\subsection{Characterization of $\mathrm{ZnO}$ nano-coated cotton fabrics}

The coated fabrics were examined in the range of basic physical-mechanical parameters followed by its functional properties (Sundaram et al 2004). The specimen were conditioned at $65 \pm 2 \%$ relative humidity and $27 \pm 2{ }^{\circ} \mathrm{C}$ before analysis. The tensile strength was carried out in Instron ${ }^{\circledR}$ Automated Materials Testing System. The bursting strength was tested on the bursting strength tester by applying the multidirectional load. Simultaneously, air permeability was tested in air permeability tester KES-F8AP1 designed by Kato Tech. Co. Ltd., Japan. Initially, the air resistance (Pa.s/m) is measured and its inverse gave the air permeability (m/Pa.s).

For friction measurements, we used an Instron ${ }^{\circledR}$ Tensile Tester fitted with an appropriate friction assembly, in accordance with the ASTM procedure (ASTM D 1984). The results were expressed as coefficient of friction $(\mu)$, which is the ratio of frictional resistance and the normal load. For UV blocking test, the UV-visible spectrometer was first calibrated using blank integrating sphere. Conditioned sample was then loaded in the integrating sphere of the instrument. Spectra were recorded directly by using the loaded integrating sphere. The results were expressed as per cent absorption of the initial UV radiation in the UV region.

\section{Results and discussion}

$\mathrm{ZnO}$ nanomaterials have some excellent properties like exceptional mechanical strength, antistatic, antibacterial and UV absorption properties (Thuenemann and Ruland 2000). UV visible spectrum taken for $\mathrm{ZnO}$ nanoparticle synthesized with $0.5 \%$ soluble starch (figure 1) shows peak absorption at $361 \mathrm{~nm}$. The energy calculated according to the Planck's constant is $3.443 \mathrm{eV}$. By using effective mass approximation, the size (diameter) was calculated to be $40 \mathrm{~nm}$.

Figure 2 shows the X-ray diffraction pattern of nano$\mathrm{ZnO}$ and bulk- $\mathrm{ZnO}$. The peaks at different crystal planes of nano- $\mathrm{ZnO}$ matches exactly with that of bulk $\mathrm{ZnO}$. The peaks assigned to diffractions from various planes correspond to hexagonal close packed structure of zinc oxide. The broadening of peaks was observed mainly due to the nano-size effect. The broad reflection at $15^{\circ}$ is due to the low crystallinity of the soluble starch. This confirms the presence of $\mathrm{ZnO}$ in the synthesized material. Presence of starch in the completely washed nano- $\mathrm{ZnO}$ indicates their strong binding nature.

To evaluate the effect of coating on fabric strength, tensile strength and bursting strength were analysed in addition to air permeability. Table 1 shows the effect of nano- $\mathrm{ZnO}$ coating on the mechanical properties of cotton fabrics. The nano- $\mathrm{ZnO}$ coating is found to reduce the tensile 


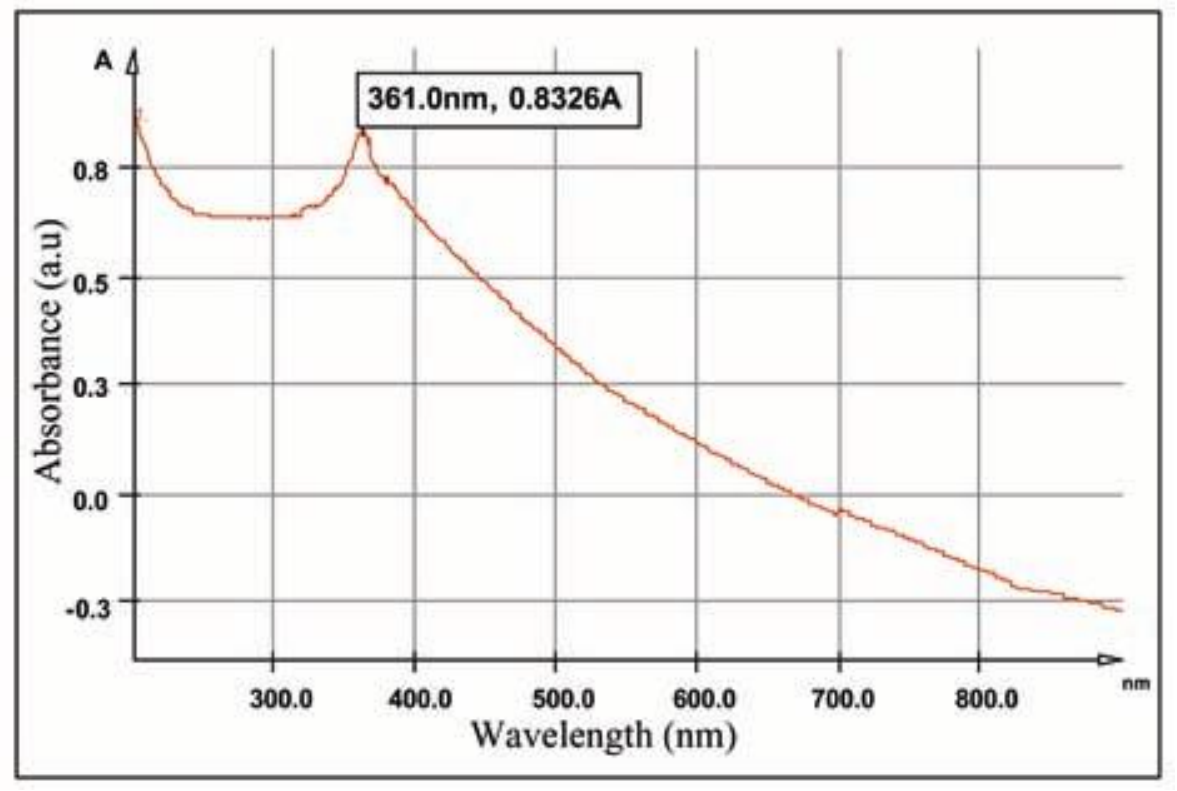

Figure 1. UV visible absorption spectrum of nano- $\mathrm{ZnO}$ solution.

Table 1. Effect of nano-ZnO treatment on mechanical properties of cotton fabrics.

\begin{tabular}{|c|c|c|c|c|c|c|}
\hline \multirow[b]{3}{*}{ Treatment on fabrics } & \multicolumn{4}{|c|}{ Tensile strength } & \multirow{3}{*}{$\begin{array}{l}\text { Air permeability } \\
{[\mathrm{m} /(\mathrm{kPa} . \mathrm{s})]}\end{array}$} & \multirow{3}{*}{$\begin{array}{l}\text { Bursting strength } \\
\qquad\left(\mathrm{kg} / \mathrm{cm}^{2}\right)\end{array}$} \\
\hline & \multicolumn{2}{|c|}{ Av. breaking load (kg) } & \multicolumn{2}{|c|}{ Strain $(\%)$} & & \\
\hline & Warp & Weft & Warp & Weft & & \\
\hline Control & $24.07 \pm 1.50$ & $21 \cdot 97 \pm 2 \cdot 12$ & $20 \cdot 05 \pm 0 \cdot 72$ & $16.07 \pm 0.88$ & $9.60 \pm 0.97$ & $6 \cdot 30 \pm 1 \cdot 04$ \\
\hline Bulk-ZnO & $24 \cdot 49 \pm 1 \cdot 19$ & $19 \cdot 78 \pm 1 \cdot 17$ & $16 \cdot 93 * \pm 1 \cdot 12$ & $12 \cdot 70 * \pm 0.04$ & $9.27 \pm 0.64$ & $6 \cdot 50 \pm 0 \cdot 61$ \\
\hline Nano-ZnO & $19 \cdot 40 * \pm 1 \cdot 25$ & $21 \cdot 61 \pm 2 \cdot 42$ & $14 \cdot 36 * \pm 4 \cdot 89$ & $13 \cdot 58 * \pm 0 \cdot 82$ & $12 \cdot 02 * \pm 1.97$ & $6.66 \pm 0.65$ \\
\hline
\end{tabular}

*Significantly different from control, at $1 \%$ level.

Table 2. Effect of nano- $\mathrm{ZnO}$ treatment on frictional properties of cotton fabrics.

\begin{tabular}{|c|c|c|c|c|}
\hline \multirow[b]{3}{*}{ Treatments on fabrics } & \multicolumn{4}{|c|}{ Coefficient of friction $(\mu)$} \\
\hline & \multicolumn{2}{|c|}{ Fabric to fabric } & \multicolumn{2}{|c|}{ Fabric to metal } \\
\hline & Warp & Weft & Warp & Weft \\
\hline Control & $1 \cdot 093 \pm 0.071$ & $1 \cdot 029 \pm 0.080$ & $0 \cdot 423 \pm 0 \cdot 010$ & $0 \cdot 442 \pm 0 \cdot 020$ \\
\hline Bulk-ZnO & $1 \cdot 324 * \pm 0.047$ & $1 \cdot 402 * \pm 0 \cdot 115$ & $0.535^{*} \pm 0.014$ & $0.515 * \pm 0.015$ \\
\hline Nano-ZnO & $1 \cdot 433 * \pm 0.091$ & $1 \cdot 072 \pm 0 \cdot 016$ & $0.491 * \pm 0.009$ & $0 \cdot 496^{*} \pm 0.008$ \\
\hline
\end{tabular}

*Significantly different from control, at $1 \%$ level.

strength of the fabric in warp direction while no significant change was noticed in the weft direction. The strain reduced significantly in both warp and weft directions. This supports the earlier finding (Sundaram et al 2004) that chemical finishes, in general, reduces the tensile strength of cotton fabrics. Air permeability is an important factor in the performance of textile materials used to provide an indication of the breathability of coated fabrics. Though the coating of fabric with bulk- $\mathrm{ZnO}$ reduces the air permeability, nano- $\mathrm{ZnO}$ coating significantly increased the same. Thus, the uniform and very thin distribution of nano- $\mathrm{ZnO}$ increased the air permeability and hence its breathability. In case of bursting strength, no significant variation was observed due to coating. 


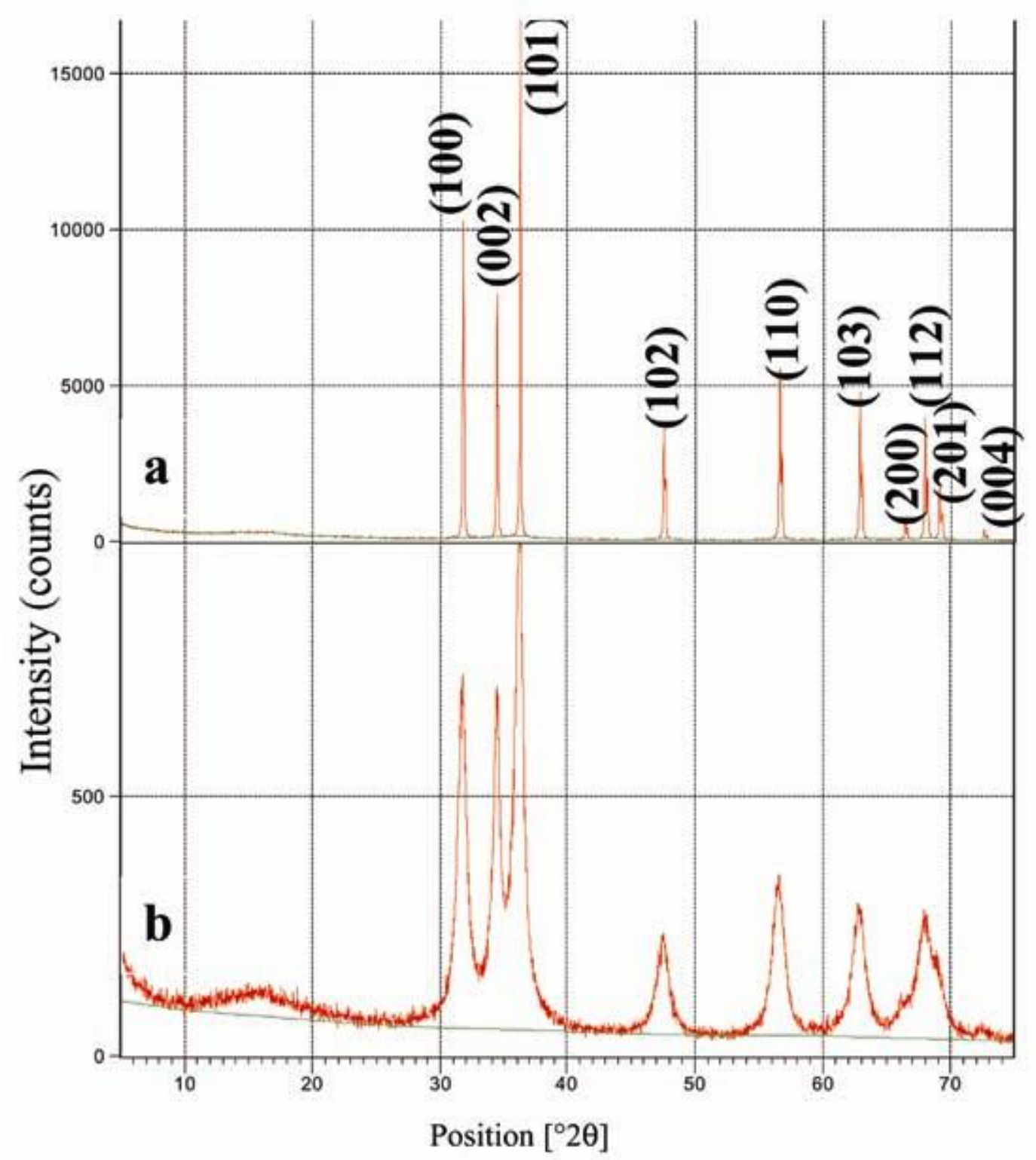

Figure 2. XRD pattern of bulk- $\mathrm{ZnO}$ (a) and nano- $\mathrm{ZnO}$ (b). The peaks assigned to diffractions from various planes are of $h c p$ zinc oxide.

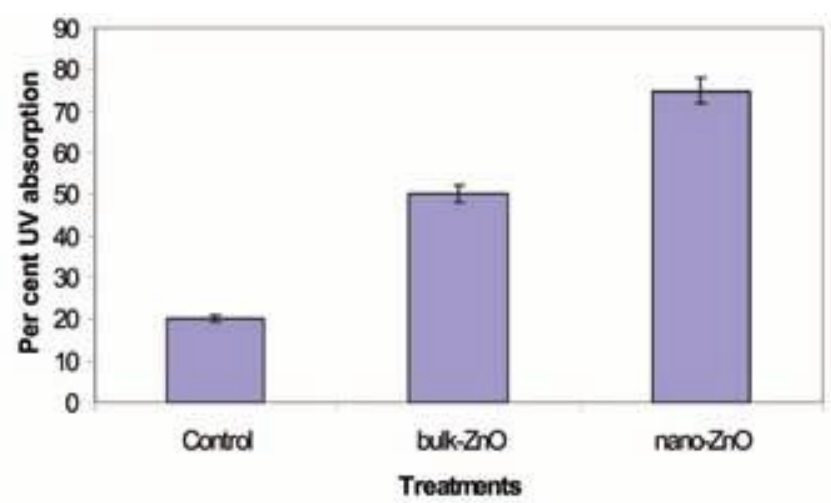

Figure 3. UV absorption of control bulk- $\mathrm{ZnO}$ and nano- $\mathrm{ZnO}$ coated fabrics.
The coefficient of friction is the parameter most frequently used to evaluate degrees of smoothness or roughness, so it is important to match this quantity with the tactile feel of the fabrics studied (Ajayi 1992). Earlier studies on friction (Basu et al 1978; Carr et al 1988; Hussain and Nachane 1998) mostly dealt with the frictional properties of cotton fibres and chemically finished fabrics. Here, we report the effect of nano-coating on cotton fabrics. Table 2 shows the effect of nano- $\mathrm{ZnO}$ coating on the frictional properties of fabric-to-fabric and fabric-tometal. The bulk- $\mathrm{ZnO}$ coated fabric resulted in significant increase in friction with both fabric and metal in comparison with control. In case of nano- $\mathrm{ZnO}$ coated fabric, due to its nano-size and uniform distribution, friction was significantly lower than the bulk- $\mathrm{ZnO}$ coated fabric. 


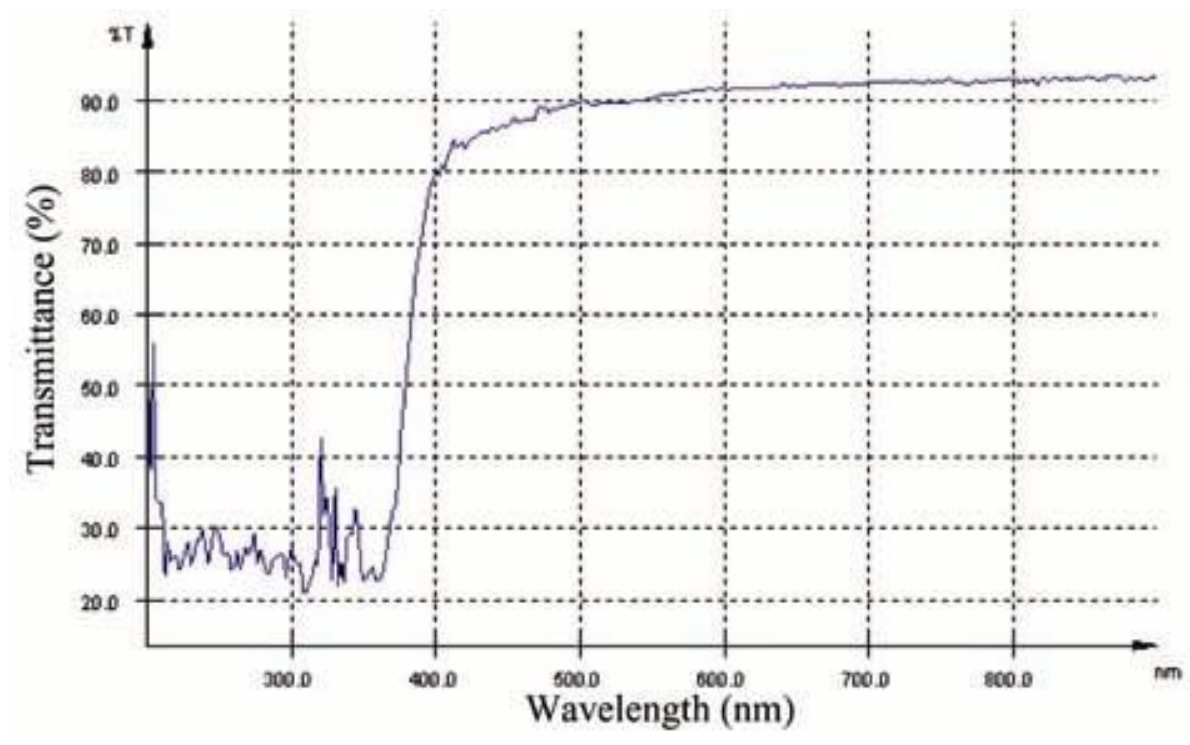

Figure 4. UV absorption spectrum of nano- $\mathrm{ZnO}$ coated fabric.

Figure 3 shows the UV absorption properties of cotton fabrics coated with nano- $\mathrm{ZnO}$. In control fabric, an average of $20 \%$ of UV light was absorbed, while the rest passed through the fabric. In case of bulk- $\mathrm{ZnO}$ coated fabric, about $50 \%$ of the UV light was absorbed by the fabric. In case of nano- $\mathrm{ZnO}$ coated fabric, a maximum of $75 \%$ absorption of UV light was noticed. The UV absorption spectrum of nano-coated fabric is shown in figure 4 . The increased surface area of nanoparticles and their uniform distribution on the fabric surface might have increased the UV absorption efficiency.

\section{Conclusions}

In conclusion, a simple method has been developed to prepare nano- $\mathrm{ZnO}$ and coat the same on cotton fabrics to impart functional properties. The nano- $\mathrm{ZnO}(2 \%)$ coated cotton fabric is found to have the UV blocking property. About $75 \%$ of the incident UV light was absorbed due to this coating. Also, air permeability of the nano- $\mathrm{ZnO}$ coated fabrics was significantly higher than the control, thus increased breathability. Nano- $\mathrm{ZnO}$ coating on cotton fabrics resulted in uniform and very thin coating due to nano-size and hence reduced friction in comparison with its bulk counterpart. Thus, the nano- $\mathrm{ZnO}$ coated cotton fabric is proved to have better strength properties, air permeability and UV-absorption property.

\section{Acknowledgements}

The authors are thankful to Dr S Sreenivasan, Director, CIRCOT, for his constant guidance and encouragement throughout the research work. Thanks are extended to Ms Anita Nair for analysis of mechanical properties.

\section{References}

Ajayi J 1992 Text. Res. J. 6252

Andres P R, Bielefeld J D, Henderson J I, Janes D B, Kolagunta V R, Kubiak P C, Mahoney J W and Osifchin G R 1996 Science 2731690

ASTM D 1984 American Society for Testing and Materials

Basu S C, Hamza A A and Sikorski J 1978 J. Text. Inst. 2/3 68

Carr W W, Posey J E and Tincher W C 1988 Text. Res. J. 3 129

Das A, Kothari V K and Vandana N 2005 AUTEX Res. J. 5133 Dickson M R and Lyon A L 2000 J. Phys. Chem. B104 6095

Hussain G F S and Nachane R P 1998 Indian Text. J. 1122

Jin H, Liu L, Gu L and Gu L 2004 Proc. 83rd TIWC, Shanghai, China p. 39

Kamat V P 2002 J. Phys. Chem. B106 7729

Lamb R, Zhang H, Jones A and Postle R 2004 Proc. 83rd TIWC, Shanghai, China p. 682

Perenboom J A A J, Wyder P and Meier P 1981 Phys. Rep. 78 173

Prasad V, Souza C D, Yadav D, Shaikh A J and Vigneshwaran N 2006 Spectrochim. Acta Part A 65173

Shanmugam S, Viswanathan B and Varadarajan T K 2005 Bull. Mater. Sci. 28629

Sundaram V, Krishna Iyer K R and Sreenivasan S 2004 Handbook of methods of tests (Mumbai: CIRCOT (ICAR))

Thomos M 1988 J. Pure Appl. Chem. 60323

Thuenemann A F and Ruland W 2000 Macromol. 331848

Wang Z L 2000 Characterization of nanophase material (Weinheim: Wiley-VCH Verlag GmbH), p. 1 